\title{
Evaluation of the Kenya Livestock and Wildlife Syndromic Surveillance System for Rift Valley Fever, Narok County, Kenya, 2018-2019
}

Erenius Nakadio ( $\square$ enakadio80@gmail.com )

Ministry of Health

Samuel Kahariri

Ministry of Agriculture

Maurice Owiny

Ministry of Health

\section{Research Article}

Keywords: Rift Valley Fever, Surveillance, Evaluation, Livestock, Wildlife, Kenya

Posted Date: October 18th, 2021

DOl: https://doi.org/10.21203/rs.3.rs-900525/v1

License: (c) (i) This work is licensed under a Creative Commons Attribution 4.0 International License.

Read Full License 


\section{Abstract \\ Background}

Rift Valley Fever (RVF) outbreaks in livestock have had a detrimental impact on livestock trade, animal breeding, and productivity. Routine evaluation and data analysis of surveillance systems ensure that health events are efficiently and effectively monitored. This study evaluated Kenya Livestock and Wildife Surveillance System (KLWSS) and characterized RVF cases reported for Narok County.

\section{Methods}

We evaluated KLWSS from January 2018 to December 2019 using CDC guidelines for evaluating surveillance systems. Attributes of simplicity, flexibility, data quality, acceptability, representativeness, timeliness, stability, sensitivity, and predictive value positive were examined. A retrospective review of RVF surveillance data for Narok County was performed. Demographic and clinical variables were assessed. Data were cleaned in Ms. Excel and descriptive analysis was done using Epi Info 7. Categorical variables were summarized using frequencies and proportions while continuous variables were summarized using measures of central tendency and dispersion. Study authorization was granted by the Directorate of Veterinary Services.

\section{Results}

System was simple in structure and operation, accommodated upgrading of its application, data quality performance was $69.8 \%$, stakeholder's participation rate was $80 \%$ with 842 reports coming from six subcounties and 30 wards. The median time between event occurrence and event reporting was two days (range one to six days). The system had been operational since 2018 with no reports of any unscheduled outages and downtimes. Suspected cases of RVF reported were 11\% (95/842) of the reported cases. The livestock species affected were cattle 56\% (53/95) and Sheep 44\% (42/95). About 96\% (91/95) of the suspected cases were in mixed livestock production systems. The common syndrome was abortions $74 \%$ (95/129) with Loita ward recording 97\% (92/95) suspected RVF cases. All suspected cases were reported in March 2018.

\section{Conclusions}

The KLWSS system was found to be stable but with below-par performance in data quality. Improvement in data quality is required to ensure that the surveillance system is efficient and effective.

\section{Background}


Rift Valley fever (RVF)is a zoonotic disease that can be transmitted to livestock and humans by mosquitoes or by direct contact with, or aerosols from, contaminated fluids and tissues[1]. The disease has been reported to be highly pathogenic in domestic livestock, specifically goats, sheep, and cattle[2]. RVF outbreaks continue to impact negatively on livestock trade, and meat and dairy industries, as infection can cause a decline in animal breeding and productivity[1].

It has been documented that economic losses resulting from the 2006-2007 RVF epidemic in Kenya were estimated at US \$32 million and in Egypt, another outbreak resulted in an estimated 200,000 human cases and 600 deaths[3].

RVF epidemics are a major global health security threat due to the high morbidity and mortality in humans, and the economic impact associated with loss of livestock and ban in international trade and most livestock RVF epidemics have been reported in Eastern Africa, occurring every 4 to 10 years and closely linked with periods of heavy rainfall that occur during the warm phase of the El Niño/Southern Oscillation phenomenon[1, 4].

Routine evaluation and data analysis of surveillance systems ensure that health events are efficiently and effectively monitored thus providing periodic recommendations for public health actions[5]. Although previous evaluations of the system have been carried out before, there is still limited evidence on how this system is performing[6].

We conducted a systematic evaluation of the Kenya Livestock and Wildlife syndromic surveillance system and also performed a descriptive analysis of the surveillance data from January 2018 through December 2019. Therefore, the findings are intended for policymakers to improve the performance of the system and for other similar settings in the country.

\section{Methods}

\section{Description of the Kenya Livestock and Wildlife Syndromic Surveillance system}

For a very long time, the animal health surveillance system in Kenya lacked a tool for real-time disease reporting[6]. With the recent experiences of rapidly emerging and spreading global outbreaks across borders and continents, there was a need to develop an effective surveillance system for Zoonotic diseases, which account for $60 \%$ of emerging diseases[7-9]. This was to be able to quickly detect and report diseases in human and animal populations to trigger timely investigations and responses. To address the above, the Centers for Disease Control and Prevention (CDC), Kenya in collaboration with the Kenya Directorate of Veterinary Services and the Kenya Wildlife Service launched the Kenya Livestock and Wildlife Syndromic Surveillance System (KLWSS). In this system, field surveillance officers submit data via a mobile application, and the data are made available to county and national authorities through a central server. Data collected by the application, called the Kenya Animal Biosurveillance System 
(KABS), are presented on a web-based dashboard with automated data analysis and feedback. KABS reports on nine syndromes i.e., abortion, sudden death, hemorrhagic, neurologic, respiratory, gastrointestinal, cutaneous, animal bites, and oral/foot lesions in seven livestock species that include cattle, sheep, goats, chicken, camels, dogs, and cats. The KABS-Wildlife form reports the same syndromes in five animal categories i.e., herbivores, carnivores, avian, aquatic, and non-human primates [6].

The stakeholders that collect and use the data generated from this system include both persons and organizations that promote public health and are involved in the prevention and control of health-related events of public health importance[6]. For example, livestock owners, health care providers (human \& livestock), county governments, national government, and non-profit organizations, and research institutions such as CDC, ILRI, and universities (Figure 1).

\section{Evaluation of the Kenya Livestock and Wildlife Syndromic Surveillance (KLWSS) System}

The surveillance system was evaluated for Rift Valley Fever across all the thirty (30) wards and six (6) sub-counties within Narok County, Kenya (Figure 2). Narok county has a livestock population of about 3.4 million (1.4 million Cattle, 1.2 million sheep, and 0.8 million goats), and residents practice both extensive and mixed livestock production systems[10].

We used the CDC guidelines to evaluate the performance of the KLWSSS in Narok county from January 2018 through December 2019. Following the CDC guidelines for evaluation of a surveillance system, nine surveillance attributes were used : (i) simplicity, (ii) flexibility, (iii) data quality and completeness for key variables, (iv) acceptability, (v) representativeness, (vi) timeliness, (vii) stability, (viii) sensitivity and (ix) predictive value positive. The attributes were evaluated as follows; Simplicity-its structure and ease of operation by users; Flexibility-Any changes in case definitions or technology; Data quality -the percentage of "unknown" or "blank" responses to items on surveillance forms; Acceptability-subjects or agency participation rate or proportion of critical variables recorded; Sensitivity-proportion of disease cases reported, the ability to detect outbreaks; Predictive value positive - the proportion of reported cases that have the health-related event under surveillance; Representativeness-how many reports were being captured in the participating sub-counties and wards; Timeliness- the time between the onset of a healthrelated event and the reporting of that event and Stability-Ability to operate since its inception[7-9, 1113]. A semi-structured and self-administered questionnaire was used to collect relevant data from surveillance officers at the county, national levels, and other stakeholders.

\section{Retrospective review of the surveillance data}

We performed a retrospective review of RVF surveillance data reported in the KLWSS system for Narok County. Any reports indicating a suspected case of livestock/wildlife presenting with acute febrile illness and/or any of the following clinical symptoms; abortion, hemorrhages, and sudden death in conformity with flooding and increased mosquito population was used as the case definition for the condition/disease of public health importance under surveillance The variables analyzed included; Ward, 
Sub- County, Date of start of event/outbreak, Date event was reported, Livestock species affected, Livestock production system type, Disease Syndromes reported (abortion, hemorrhagic syndromes, sudden death). The following Key variables; Date event was reported, nature of the diagnosis, laboratory test, abortion, hemorrhages, the number at risk, the number affected/sick, and number dead were used to evaluate the completeness of data in the surveillance database with a lower and upper threshold set at $80 \%$ and $90 \%$ respectively and the upper threshold was considered complete.

\section{Data Collection, Management, and Analysis}

Data were extracted from the reports in the KLWSS system and exported in Ms. Excel spreadsheets. The following variables were analyzed; Ward, Sub- County, Date of start of event/outbreak, Date event was reported, Livestock species affected, Livestock production system type, Disease Syndromes reported (abortion, sudden death, hemorrhagic). Data were cleaned, audited for quality using the Data Quality Audit (DQA) tool, and stored in password protection. Categorical variables were summarized using frequencies and proportions while continuous variables were summarized using Measures of Central tendency and dispersion. Analyzed data was presented in tables and graphs.

\section{Ethical Considerations}

The Kenyan Ministry of Agriculture, Livestock, Fisheries, and Cooperatives considers that syndromic surveillance for Rift Valley Fever is a part of routine public health surveillance, and therefore did not require formal ethical review because data reported are used for public health surveillance services. Data were anonymized upon collection, and authors did not have access to identifying information since data was stored in password-protected excel sheets. Data abstraction was authorized by the National Director of Veterinary Services (DVS).

\section{Results}

\section{Evaluation of the surveillance system}

\section{System Attributes}

\section{Simplicity}

From the time of establishment to the period of evaluation, the system had recorded 842 reports and 95 suspected cases of RVF in Narok county (Figure 3). The animal health service providers who underwent training at the beginning continued to report diseases to the veterinarians who then make diagnoses, treatment, and report disease cases to the system in near real-time. No refresher training had been conducted but the data continued to be collected. A $100 \%$ of the users reported that it was easy to operate the mobile application.

\section{Flexibility}


The users of the system reported that following its implementation, there were issues on both the location and the syndromic information sections but after continuous upgrading of the KABs application, those challenges were resolved. The ease to accommodate those changes reflected the flexibility of the surveillance system to respond to change quickly.

\section{Data quality}

A total of 51 variables were in the surveillance system database. About $33(65 \%)$ were relevant to the condition under surveillance. From the Data quality Audit Tool,698 YES and 302 NOs were generated with an overall performance of $69.8 \%$ for the surveillance data quality. Some variables did not meet the lower threshold of $80 \%$ set for data quality assessment and analysis (Figure 5).

\section{Acceptability}

The system was being used by all the relevant stakeholders within the county and the country, with a stakeholder's participation rate of $80 \%$. All the six (6) sub-counties and 30 wards in Narok county had reported cases during the review period. All the critical variables within the system had appropriate values recorded (100\%).

\section{Sensitivity}

For the period of the evaluation, the system reported 95 (ninety-five) suspected cases of RVF $(11 \%$ of total suspected cases reported). All 95 cases of RVF were reported in the year 2018 and the month of March. Although this attribute gave the proportion of suspected RVF cases in the study population, it was not certain whether they were true cases or not due to a lack of confirmatory laboratory data, a limitation for this evaluation.

\section{Predictive Value Positive}

This attribute was not assessed because the system was not able to identify the actual number of RVF cases or deaths caused by RVF infection among the general livestock and wildlife population in Narok county due to a lack of the laboratory data that could have been used to reflect the actual situation of the health-related event. This was identified as one of the limitations of the surveillance system.

\section{Representativeness}

All the 842 records in the surveillance system came from all the six (6) sub-counties and 30 wards in the county. $95 \%$ of the RVF suspected cases were reported from the Loita ward in the Narok South subcounty.

\section{Timeliness}

The median time between the date of occurrence of the disease event and reporting of the event in the system was two (2) days with a range of one (1) to six (6) days. 


\section{Stability}

The users reported that the system had been operational since March 2018 and there were no reports of any unscheduled outages and downtimes for the system's software and hard wares being hosted at the offices of the National Directorate of Veterinary Services.

\section{Descriptive Analysis of surveillance system data}

For the period of the evaluation,842 records of livestock syndromes were reviewed and analyzed. Out of the total records, the surveillance system reported 95 suspected cases of RVF (11\%) (Figure 3). The livestock species affected were Cattle at 56\% (53) and Sheep at 44\% (42) and many suspected cases were in the mixed livestock production system areas at $96 \%$ (91) (Table 1). The commonly reported syndrome was abortions 95 (73\%) with the clinical diagnosis being the only means of diagnosis at $100 \%$ (95) (Table 2, Figure 4). Loita ward in Narok South sub-county reported most cases of RVF at 97\% (92) during the evaluation period (Table 1). All the 95 RVF suspected cases were reported in the year to March 2018 (Table 1).

Table 1: RVF Demographic Data, Narok County, 2018-2019

$(n=95)$

\begin{tabular}{llll} 
Variable & & Frequency(n) & Proportion (\%) \\
\hline Livestock Species & Cattle & 53 & 56 \\
\hline \multirow{2}{*}{ Livestock Production systems } & Sheep & 42 & 44 \\
\hline \multirow{2}{*}{ Year } & Mixed & 91 & 96 \\
\hline \multirow{2}{*}{ Ward } & Extensive & 4 & 4 \\
\hline \multirow{3}{*}{ Sub County } & 2018 & 95 & 100 \\
\hline \multirow{3}{*}{} & 2019 & 0 & 0 \\
\hline & Loita & 92 & 97 \\
\hline & Mara & 2 & 2 \\
\hline & Mosiro & 1 & 1 \\
\hline & Narok South & 92 & 97 \\
\hline & Narok West & 2 & 2 \\
\hline & Narok East & 1 & 1
\end{tabular}


Table 2

Clinical Characteristics of RVF cases, Narok County, 2018-2019

\begin{tabular}{|llll|}
\hline Variable & & Frequency(n) & Proportion (\%) \\
\hline Livestock Species & Cattle & 53 & 56 \\
\hline Livestock Production systems & Sheep & 42 & 44 \\
& Mixed & 91 & 96 \\
\hline Year & Extensive & 4 & 4 \\
\hline & 2018 & 95 & 100 \\
\hline Ward & 2019 & 0 & 0 \\
\hline & Loita & 92 & 97 \\
\hline Sub County & Mara & 2 & 2 \\
\hline & Mosiro & 1 & 1 \\
\hline & Narok South & 92 & 97 \\
\hline (n=95) & Narok West & 2 & 2 \\
\hline
\end{tabular}

\section{Discussion}

This evaluation demonstrated that the KLWSS system was simple in structure, easy to operate by the users, and accommodated the upgrading of its KAP application. Although there was active participation and use of the system by key users and stakeholders, the data quality was below par. The median time between event occurrence and event reporting was within the acceptable range and the surveillance system had been operational since 2018 with no reports of any unscheduled outages and downtimes. Descriptive analysis of the surveillance data revealed that cattle and sheep were the most affected especially in mixed livestock production systems. The most commonly reported syndrome was abortion and most suspected cases of RVF were from the Loita ward, mainly in 2018 compared to 2019.

The KLWSS system was useful because it reported a majority of RVF suspected cases in Narok county. In the Kenya Animal Biosurveillance system (KABS) application, a straightforward case definition based on syndromes was being used to classify the cases illustrating the simplicity of the system. The system was flexible as it accommodated slight changes during the upgrading of the KABs application and very little additional time and resources were injected during the application upgrade. The RVF data for Narok county was of below-par performance in terms of quality since not all the critical variables had 
appropriate values recorded for them. This could be attributed to technological challenges and a shortage of trained staff in most surveillance sites.

Multiple stakeholders were utilizing the system for reporting and all the participating sub-counties and wards within Narok county had at least reported suspected RVF cases during the review period.

Timeliness was one of the system's major strong points because there was a short time between the occurrence of the disease and reporting of the event in the system. This means that the system can report events under surveillance in near real-time.

The livestock species most affected were Cattle and Sheep in mixed livestock production system areas. These findings were consistent with the outbreak of June 2018 that occurred in Wajir and Marsabit counties where it was reported that the disease was highly pathogenic in cattle, sheep, and goats $[14,15]$. The most commonly reported syndrome was abortions compared to other syndromes of abortions/hemorrhages or abortions/sudden deaths. Other studies have also documented nearly related findings[16, 17]. Increased frequency of the disease in March 2018 could be a true outbreak or a result of other factors such as prompt reporting or even the fact that the system was launched the same month and year hence the increased cases reporting in that period. Further investigations should be carried out to ascertain whether the increase in RVF suspected cases in march 2018 in Narok county was a true outbreak or not. Loita ward in Narok South sub-county reporting most cases could likely be due to highest population of livestock species there or aggressiveness of the frontline animal health officers in that ward or there could be other motivating factors. Further analysis of the data from this location could provide more evidence.

This evaluation was subject to at least two limitations. Firstly, sensitivity and predictive value positive (PVP) attributes of the system were not assessed because of a lack of data on the actual number of cases among the population. Secondly, the cause of death for the disease under investigation could not be independently ascertained to be attributed to it or could be as a result of other diseases/conditions whose syndromes are also reported in the system. These limitations made it difficult to compute the critical measures of disease frequency especially morbidity and mortality rates which would have assisted the users of the system to detect any outbreak of the disease event under investigation.

\section{Conclusions}

The KLWSS system was stable, simple to use, and highly acceptable but with below-par performance in data quality. However, it was limited in the detection of outbreaks since there was no baseline data available. Therefore, improvement in data quality is required to ensure that the system was efficient and effective in its performance. More data should be collected to inform and promote prevention interventions especially among the most vulnerable livestock species in the county and country.

\section{Declarations}




\section{Ethics approval and consent to participate}

Ethical approval was not required for this study since the Kenyan Ministry of Agriculture, Livestock, Fisheries, and Cooperatives considers that syndromic surveillance for Rift Valley Fever is a part of routine public health surveillance. Participation in the study was voluntary and submission of the completed questionnaire was considered consent to participate. The confidentiality of participant identity was maintained and all data was aggregated to ensure the responses from specific participants could not be identified. All the methods used for this study were performed in accordance with the relevant guidelines and regulations.

\section{Consent for publication}

Not applicable

\section{Availability of data and materials}

The data that support the findings of this study are available from National Directorate of Veterinary services registry but restrictions apply to the availability of these data, which were used after approval for the current study, and so are not publicly available. Data from this registry are however available from the authors upon reasonable request and with permission of the National Director of Veterinary services.

\section{Competing interests}

None

\section{Funding}

There was no external or additional funding for this study. However, the study was supported by the Center for Disease Control and Prevention (CDC), Kenya through the Kenya Field Epidemiology and Laboratory Training Programme (KFELTP) of the Kenyan Ministry of Health.

\section{Authors' contributions}

All authors have read and approved the manuscript and take responsibility for the integrity of the data and the accuracy of the data analysis. Study concept and design: EN, SK, MO, Acquisition, analysis or interpretation of data: EN, Drafting of the manuscript: EN, MO.

\section{Acknowledgements}

We thank the Kenya government animal health officers at the county level for providing important information during the evaluation. The willingness of the senior leadership at the Kenya Directorate of Veterinary Services to grant permission for us to access the surveillance data. The evaluation was supported by US Center for Disease Control and Prevention (CDC) under the Field Epidemiology and Laboratory Training Programme-Kenya Office. 


\section{Disclaimer}

The findings and conclusions in this article are those of the authors and do not necessarily represent the official position of the United States' Centers for Disease Control and Prevention.

\section{Authors information}

${ }^{1}$ Field Epidemiology and Laboratory Training Program, Ministry of Health, Kenya

${ }^{2}$ Directorate of Veterinary of Services, Turkana County Government, Kenya

${ }^{3}$ Veterinary Epidemiology and Economics Unit, Directorate of Veterinary Services, Ministry of Agriculture, Livestock, Fisheries and Cooperatives, Kenya

\section{References}

1. WHO Report 2018. Introduction to Rift Valley Fever.:1-22.

2. Poueme R, Stoek F, Nloga N, Rissmann M, Schulz A, Wade A, et al. Seroprevalence and Associated Risk Factors of Rift Valley Fever in Domestic Small Ruminants in the North Region of Cameroon. 2019;2019.

3. Nanyingi MO, Munyua P, Kiama SG, Muchemi GM, Thumbi SM, Bitek AO, et al. A systematic review of Rift Valley Fever epidemiology 1931 2014. 2015;1:1-12.

4. Oyas H, Holmstrom L, Kemunto NP, Muturi M, Mwatondo A, Osoro E, et al. Enhanced surveillance for Rift Valley Fever in livestock during El Niño rains and threat of RVF outbreak, Kenya, 2015-2016. PLoS Negl Trop Dis. 2018;12:2015-6.

5. Mariner 2018. Rift Valley Fever Surveillance.

6. Njenga MK, Kemunto N, Kahariri S, Holmstrom L, Oyas H, Biggers K, et al. High real-time reporting of domestic and wild animal diseases following rollout of mobile phone reporting system in Kenya. bioRxiv. 2020.

7. $10.1371 /$ journal.pone. 0161576 [doi].

8. Munyua P, Bitek A, Osoro E, Pieracci EG, Muema J, Mwatondo A, et al. Prioritization of zoonotic diseases in Kenya, 2015. PLoS One. 2016;11:1-11.

9. Dureab F, Ahmed K, Beiersmann C, Standley CJ, Alwaleedi A, Jahn A. Assessment of electronic disease early warning system for improved disease surveillance and outbreak response in Yemen. BMC Public Health. 2020;20:1-11.

10. Siaya CIDP 2018. County integrated development plan. 2018.

11. Yazidi R, Aissi W, Bouguerra H, Nouira M, Kharroubi G, Maazaoui L, et al. Evaluation of the influenzalike illness surveillance system in Tunisia, 2012-2015. BMC Public Health. 2019;19:1-9.

12. Babakazo P, Kabamba-Tshilobo J, Wemakoy EO, Lubula L, Manya LK, Kebela llunga B, et al. Evaluation of the influenza sentinel surveillance system in the Democratic Republic of Congo, 2012- 
2015. doi:10.1186/s12889-019-8008-2.

13. CDC. Field Guidelines. Overview of Evaluating Surveillance Systems. 2013;:18. doi:10.1523/JNEUROSCI.0974-04.2004.

14. Hassan A, Muturi M, Mwatondo A, Omolo J, Bett B, Gikundi S, et al. Epidemiological Investigation of a Rift Valley Fever Outbreak in Humans and Livestock in Kenya, 2018. 2020;:1-7.

15. Mohamed AM, Ashshi AM, Asghar AH, Abd El-Rahim IHA, El-Shemi AG, Zafar T. Seroepidemiological survey on Rift Valley fever among small ruminants and their close human contacts in Makkah, Saudi Arabia, in 2011. Rev Sci Tech. 2014;33:903-15.

16. Jost CC, Nzietchueng S, Kihu S, Bett B, Njogu G, Swai ES, et al. Epidemiological Assessment of the Rift Valley Fever Outbreak in Kenya and Tanzania in 2006 and 2007. 2010;83 Suppl 2:65-72.

17. Budasha N, Gonzalez J, Sebhatu T, Arnold E. Rift Valley fever seroprevalence and abortion frequency among livestock of Kisoro district, South Western Uganda (2016): A prerequisite for zoonotic infection. j. 2018;14.

\section{Figures}

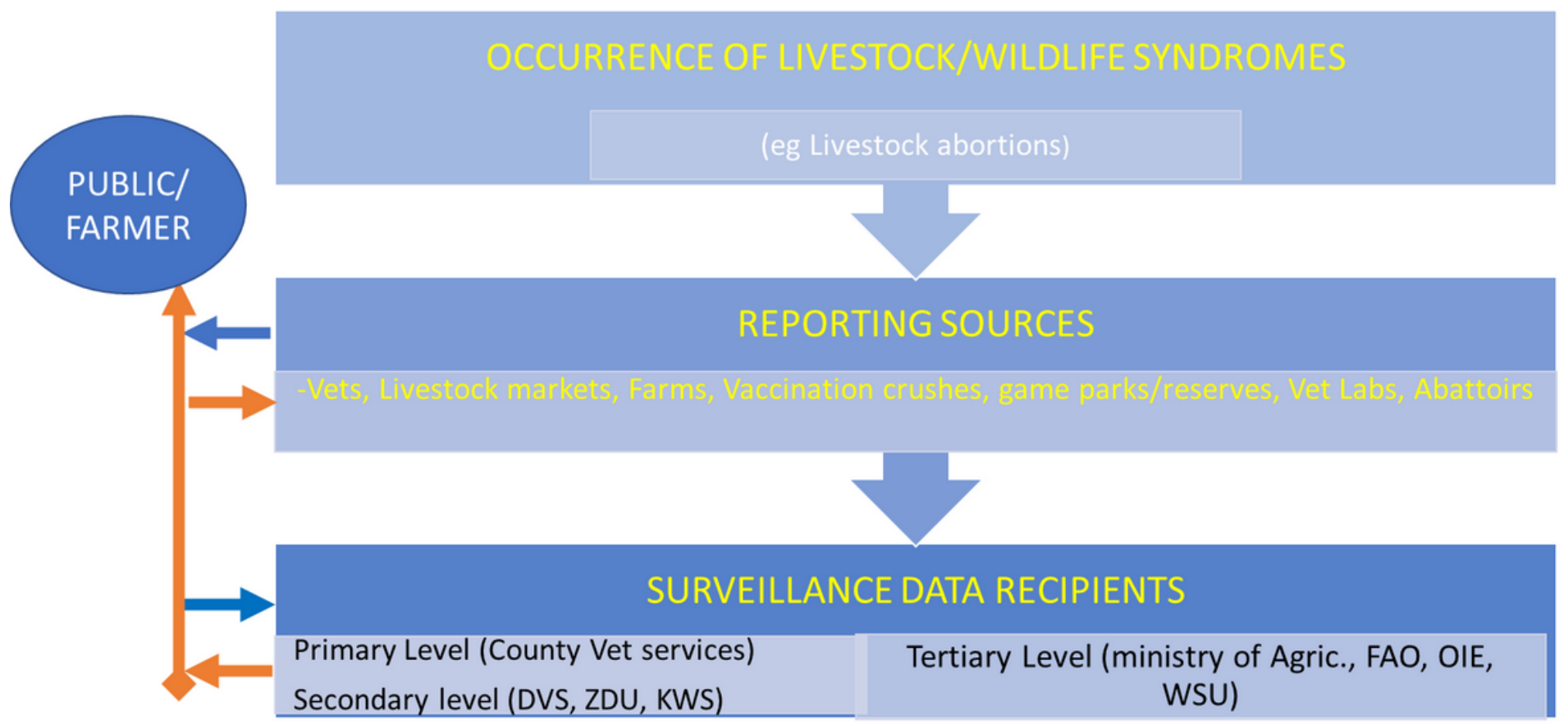

\section{Figure 1}

Data flow and feedback within the KLWSS system; DVS Report,2018 


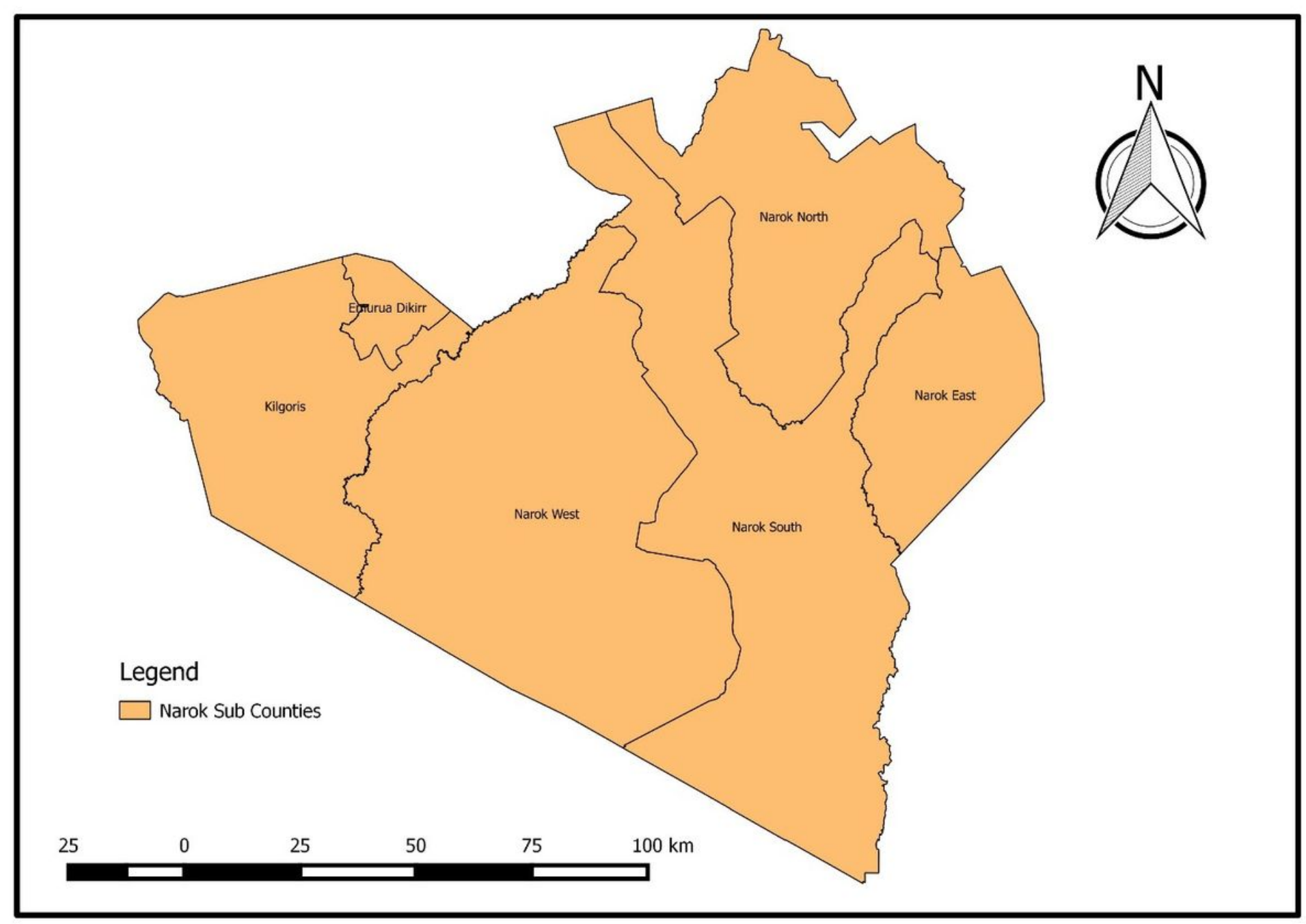

Figure 2

Location of the Surveillance Sites in Narok County, Kenya 


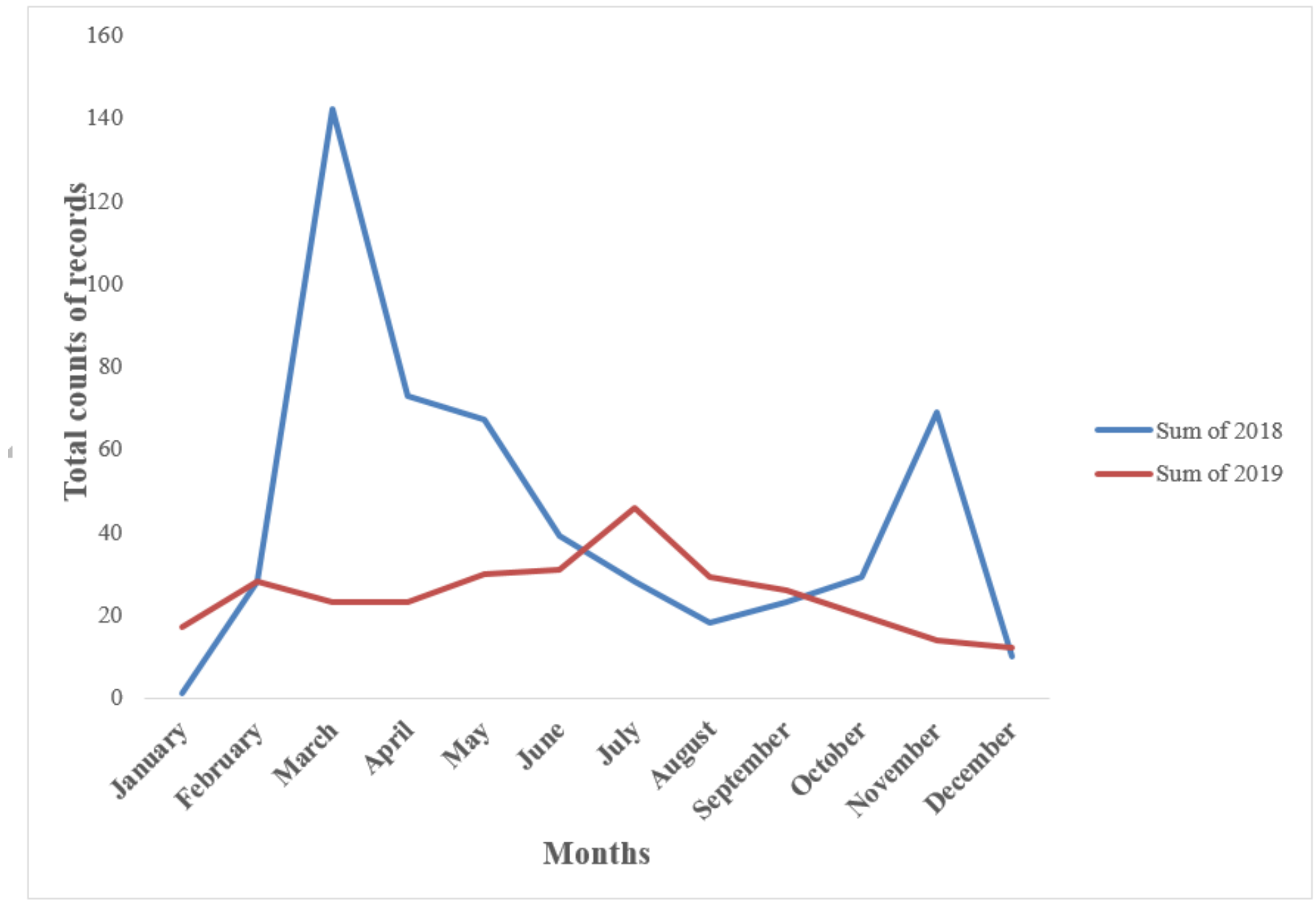

\section{Figure 3}

Total Count of Records in Narok County, 2018-2019 


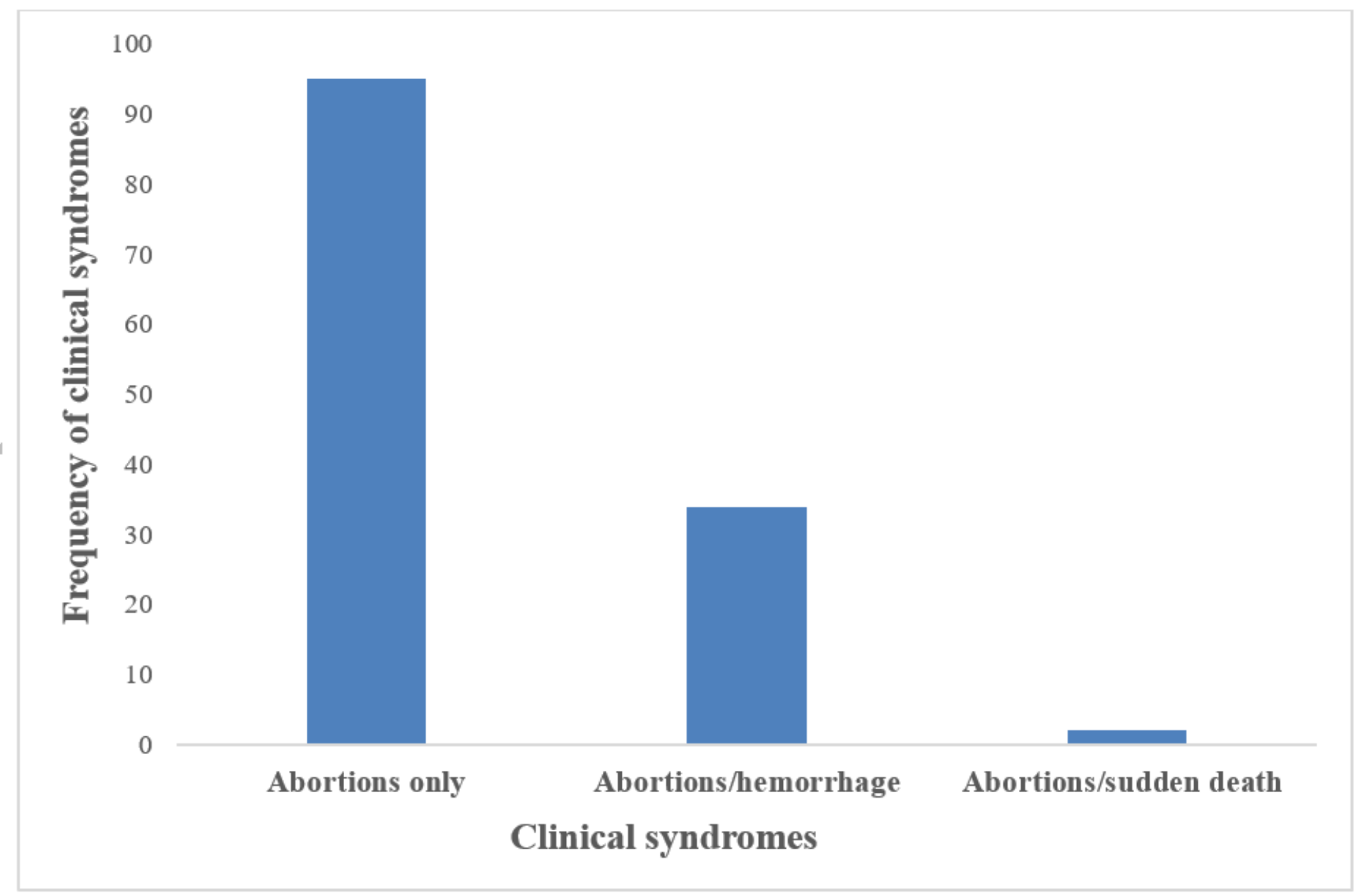

Figure 4

Frequency of commonly reported syndromes, Narok County,2018-2019 


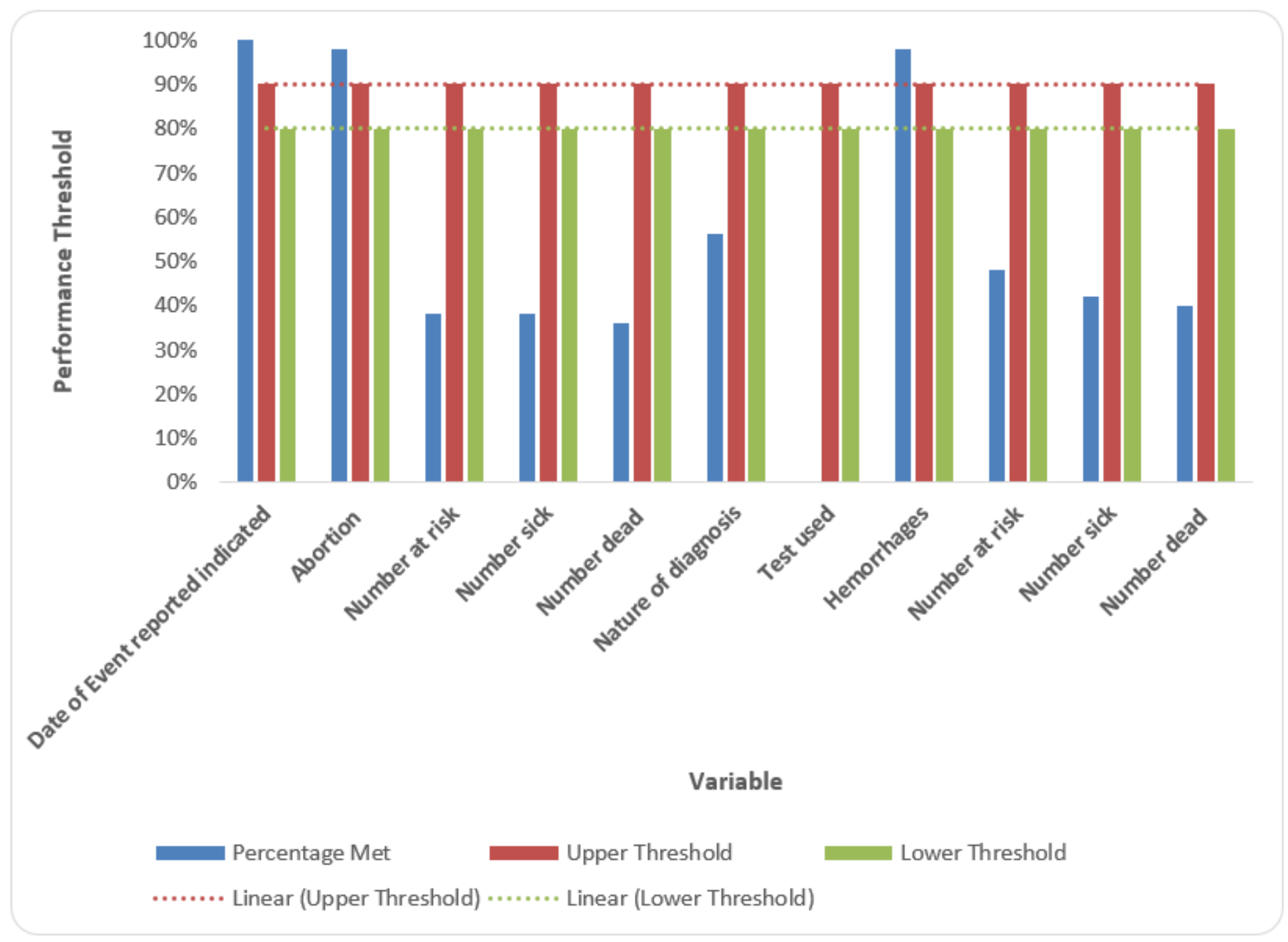

Figure 5

Data Audit Tool of select data variables for RVF cases, Narok County, 2018-2019 